\title{
Prior experience and complex procedures
}

\author{
PETER DIXON, CORINNE ZIMMERMAN, and SIOBHAN NEAREY \\ University of Alberta, Edmonton, Alberta, Canada
}

\begin{abstract}
The role of prior knowledge in learning complex procedures was investigated in a transfer task in which subjects learned two related procedures in sequence. In Experiment 1, we manipulated the conceptual and structural similarity between the two procedures; in Experiment 2, we manipulated whether the order of the steps within subprocedures was the same or different during training and transfer, or whether the order of the subprocedures was the same or different. The results lead us to hypothesize that transfer in complex procedures is mediated primarily by a memory for specific steps rather than by conceptual understanding or problem solving. In particular, we were able to model the results precisely on the assumption that subjects use superficial similarity to retrieve the sequences of steps needed to perform segments of the procedure.
\end{abstract}

People successfully perform a variety of complex procedures every day, ranging from relatively mundane activities such as baking a cake or balancing a checkbook, to more technology-dependent procedures such as programming a VCR or using a fax machine. And although there are a few notorious counterexamples, even novel procedures are generally learned quickly with little apparent effort. For example, cooks typically can use a new recipe with ease, and a new model of banking machine usually poses no problem to a user. Our view is that the success that is typically enjoyed in such situations is a function of prior knowledge: Novel procedures are acquired easily when people are already familiar with similar procedures. In the present research, we were concerned with the nature of this prior knowledge and how it is used in acquiring new procedures.

Previous research has suggested that two kinds of knowledge might be involved in learning new procedures: conceptual knowledge and structural knowledge. Conceptual knowledge refers to information about why a procedure works, what a device does, or how it operates. For example, in learning to operate a VCR, relevant conceptual knowledge might include the fact that information is recorded on magnetic tape in the cassette, that the tape is transported from the one side of the cassette to the other during playing and recording, that VCR operations can be controlled by a programmable timer, and so on. On the other hand, structural knowledge refers to knowledge about the structure of the procedures: the steps that have to be performed, the order of the steps, their organization into subtasks, and the rules that determine legal sequences of steps. For example, structural knowledge regarding VCRs

This research was supported by a Natural Sciences and Engineering Research Council of Canada research grant to the first author. Siobhan Nearey is now with the Department of Psychology, Carnegie Melion University, Pittsburgh, PA 15213. Correspondence should be addressed to P. Dixon, Department of Psychology, University of Alberta, Edmonton, AB, Canada T6G 2E9 (e-mail: pdixon@psych.ualberta.ca). might include the fact that there are procedures for setting the time, selecting channels, and programmed recording, that there are buttons for moving to and from an on-screen programming mode, and so on.

Conceptual knowledge can be distinguished from structural knowledge by virtue of its relationship to world knowledge beyond the specific context of the device or procedure at hand. In particular, conceptual knowledge about a device or procedure is embedded in a field of more general conceptual information about the world. Because conceptual knowledge is based on more general world knowledge, it is often useful in troubleshooting. For example, suppose that one's VCR does not play because there is a general power outage in the building. One might be able to diagnose such a problem by combining the fact that the VCR requires power (conceptual knowledge about the device) with the fact that no power is available in the building (inferred from the observation that none of the lights and other electrical appliances are working either). Central to this reasoning process is the fact that the concept of "power" involved in the idea "VCRs require power" is connected to a wealth of information about electrical appliances in the real world. Without this connection, the diagnosis of this particular problem would be much more difficult.

On the other hand, we assume that structural knowledge makes relatively little contact with broader conceptual or semantic information; instead, we view it as limited to a particular device or procedure. Consequently, structural knowledge is less likely to support chains of inferences involving world knowledge. For example, suppose that the television is showing static rather than the recorded signal from the VCR because the television has not been tuned to the correct channel. A user is likely to have knowledge that is relevant to this difficulty; for example, one might know that the television must be set to Channel 4 in order for one to view a tape. However, for some individuals, this fact could be disconnected from any knowledge about how the device works and consequently would 
constitute structural knowledge rather than conceptual knowledge. The result is that the information would be unhelpful in diagnosing why the VCR is not operating correctly and would not directly support any specific inference about how to remedy the problem. One might be able to use a general heuristic, such as, "If the VCR is not operating, it has not been set up correctly." One could then successfully solve the problem by rerunning the entire set up procedure, including setting the television channel to 4 . Crucially, however, the user would be unable to use more general knowledge to infer the nature of the difficulty.

In our usage, the defining characteristic that determines whether knowledge should be classified as conceptual or structural is the relationship to general world knowledge: Conceptual knowledge refers to and depends on concepts and semantic information that apply to the world in general, whereas structural knowledge only involves features and aspects of the task at hand. Consider the knowledge that users have of banking machines. Some of this knowledge is conceptual: For example, users know that withdrawing cash from their account causes the account balance to be debited. This knowledge is conceptual because it refers to concepts such as cash, bank accounts, and balances, all of which are used in a wide range of other contexts in the world. Other knowledge seems more structural: For example, users may know that numerical entries are always terminated by pressing the "OK" button. This knowledge is structural because terms such as "numerical entry" and "OK button" only have a clear meaning in the particular context of operating banking machines and because the knowledge is of little relevance beyond that context.

One diagnostic of the distinction between conceptual and structural knowledge is generality. Conceptual knowledge is likely to be relevant across a wide range of different procedures and devices that perform similar functions. For example, conceptual knowledge concerning withdrawals and bank balances applies regardless of whether one uses a banking machine to perform a transaction or whether the transaction is carried out over the counter. Similarly, much of the conceptual knowledge about playing and recording signals on a VCR is also relevant to audio cassette recorders and reel-to-reel tape recorders. On the other hand, structural knowledge of a procedure or device only applies to superficially similar procedures and devices. For example, information about when to press "OK" may be relevant to other banking machine models, but it is unlikely to apply to entirely different methods of withdrawing money from a bank account. Similarly, structural knowledge about how to get into and out of a VCR's on-screen programming mode may be relevant for similar VCRs, but it would not apply to early-model VCRs without on-screen programming and would not apply to audio tape recorders.

The distinction between conceptual and structural knowledge is not always clear cut, and some of the knowledge that is relevant to a procedure may have both structural and conceptual aspects. For example, users' knowledge about the role of personal identification numbers in the use of banking machines may be largely structural: Users may view their number as simply a component of the procedure required to access their bank accounts with no connection to more general world knowledge. On the other hand, users may also view a personal identification number as analogous to a signature on a check or similar to a key used to open a safe. Although such analogies are not perfect, they are clearly conceptual because they involve concepts that apply to a wide range of situations in the world. Thus, it seems likely that knowledge about personal identification numbers is neither purely conceptual nor purely structural. We do not view ambiguity of this sort as a problem for our analysis. The purpose in drawing a distinction between conceptual and structural knowledge is not to imply that all knowledge is either one or the other, but rather to consider the effects of different types of knowledge on the acquisition of new procedures; in particular, we are interested in the hypothesis that knowledge that is clearly conceptual has different effects on the acquisition of new procedures than does knowledge that is clearly structural. Presumably, realworld knowledge that has both conceptual and structural aspects would display a mixture of such effects.

Previous research has demonstrated the importance of both conceptual and structural knowledge. A clear demonstration of the value of conceptual knowledge was provided by Kieras and Bovair (1984). Subjects were asked to manipulate a control panel in order to make a particular light flash four times. One group of subjects was told that the control panel represented the phaser bank controls for the Starship Enterprise and were given detailed information about the internal connections and operating principles of the device; a control group was not given this information. The "Star Trek" group learned and executed the procedures faster, had better retention of the materials and procedures, and generated more short-cuts than did the control group. Kieras and Bovair concluded that conceptual information about the device improved performance because it allowed subjects to make inferences about the device and its operating procedure. Similar benefits of conceptual knowledge have been found by Bayman and Mayer (1984) and Halasz and Moran (1983), among others.

Previous research has also demonstrated the advantage of structural knowledge. For example, Dixon and Gabrys (1991) asked subjects to learn to operate two devices, one after the other. Performance with the second device was substantially better when its operating procedure was structurally similar to that for the first device. Moreover, this transfer occurred regardless of whether the devices were conceptually similar: The amount of transfer was almost as large when the first device was an airplane and the second was a video disk player as when two types of airplanes were used. Dixon and Gabrys concluded that the transfer occurred because an identical organization was used in the two tasks to group steps into subtasks. Similar conclusions were reached by Kamouri, Kamouri, and Smith (1986). After learning several different procedures, subjects produced substantial transfer to new 
tasks with formally identical structures. As in the research by Dixon and Gabrys, this transfer was obtained even when there was little conceptual relation between the procedures. However, unlike Dixon and Gabrys, Kamouri et al. found effects of structural similarity only when subjects learned the procedures in an "exploratory" manner, without written instructions. A related result was reported by Smith and Goodman (1984): They found that subjects carried out an assembly task more effectively when they were given a general organizational principle such as, "Assembling a circuit requires that you get the major components ready, then connect them."

Although these and other studies have conclusively demonstrated the value of structural and conceptual knowledge, the circumstances under which one kind of knowledge or the other is used have not been clearly delineated. In the present work, we evaluate the hypothesis that the utility of conceptual and structural knowledge depends on the nature of the steps that have to be learned: The acquisition of some steps in a procedure may be mediated primarily by prior conceptual knowledge, whereas the acquisition of other steps may be mediated primarily by prior structural knowledge. We refer to steps as content steps if they can be readily interpreted in terms of what the task accomplishes or how the device works. For example, pressing the "play" button on a VCR is a content step, because it has an obvious interpretation in terms of the movement of tape in the cassette, the internal generation of video signals, and so on. It seems likely that conceptual knowledge could be used to anticipate when content steps would have to be performed, and consequently, prior conceptual knowledge should aid in the acquisition of content steps in a procedure.

We refer to other steps, which are less easily interpreted in terms of the operation of the device, as formal steps. For example, pressing the "mode" button to initiate a procedure for setting the clock would be a formal step. Although users may know that such a step has to be performed, it is unlikely that they could articulate any function for the step in terms of the underlying concepts or principles of operation. Thus, conceptual knowledge is unlikely to help in learning formal steps because formal steps cannot be predicted from knowledge of how the device works. However, structural knowledge of similar devices might help. For example, users might be able to predict that the "mode" button needs to be pressed because a similar button must be pressed to initiate procedures on other types of VCRs. We refer to the idea that conceptual knowledge aids acquisition of content steps while structural knowledge aids acquisition of formal steps as the hypothesis of step-specific transfer.

One diagnostic for the distinction between content and formal steps is based on users' understanding of why a given step is necessary. If users can generate an explanation for a step in terms of concepts and relationships that apply to a wider range of situations than the particular device or procedure, that step is considered a content step in our terminology. On the other hand, if users can only explain a step in terms of the other elements of the procedure, that step would be considered a purely formal step. For example, when asked why one has to press an account button when withdrawing cash from a banking machine, users would likely be able to generate an explanation such as, "The machine needs to know which account to withdraw money from." Because this explanation is cast in terms of concepts such as money and accounts, which apply to a wide range of situations beyond the particular procedure, this step would be classified as a content step. However, in the same context, when asked why one has to press "OK" after entering an identification number, users may only be able to provide explanations such as, "It's required to confirm the input," or "It has to be pressed before going on to the next step." Because these kinds of explanations refer only to the procedure and the device, rather than to more general concepts, that step would be classified as a formal step.

As we have defined it, the distinction between content and formal steps is not hard and fast, and the classification of a step may vary with the knowledge and background of the user. For example, the step of tuning a television to Channel 4 in order to view a videotape may be a purely formal step for some because it is unrelated to any knowledge of how VCRs and televisions operate. In particular, such individuals would be unable to generate a deep explanation of why the step is necessary. However, other users may have some understanding that the VCR generates video signals at a particular frequency and that the television needs to be tuned to that frequency. For these individuals, the step of tuning the television to Channel 4 would be a content step. In the present experiments, subjects were presented with novel devices, and the information provided about each step in the procedures was deliberately manipulated. Thus, some of the steps were unambiguously content steps because subjects were given a simple interpretation of the step in terms of the function of the device. Our assumption is that subjects would be able to articulate an explanation for these steps that involved conceptual knowledge beyond the particular device. Other steps were unambiguously formal steps because there was no simple interpretation of the function of that step. Thus, it is unlikely that subjects would be able to generate any explanation of why the step was required in the procedure.

\section{EXPERIMENT 1}

In the first experiment, a transfer paradigm was used to test the hypothesis of step-specific transfer. Subjects were asked to learn an operating procedure for a device to criterion and were then asked to learn to operate a second, related device. The second device was either conceptually similar to the initial device or structurally similar. Our hypothesis was that when the devices were conceptually similar, the conceptual knowledge gained during the acquisition of the first procedure would aid in the acquisition of the new content steps. On the other hand, when the operating procedures were structurally similar, the structural knowledge learned during the acquisition 
of the initial device should aid in the acquisition of the new formal steps.

\section{Method}

Subjects first learned to operate a training device and then learned to operate a transfer device. The transfer device was either conceptually similar or dissimilar and either structurally similar or dissimilar to the training device, yielding four between-subjects conditions.

Apparatus and Materials. The devices were simulated on the monitor of a Macintosh computer. Standard Macintosh dialog boxes were used to represent each device; the dialog box for each device contained 13 buttons and two lights. On some steps, subjects were required to "press" a button on the simulated device by clicking the mouse at the location of the button. On other steps, subjects were required to wait from 1 to $2 \mathrm{sec}$ for a designated light to flash for a half second. A light consisted of a circle that was either filled (on) or unfilled (off).

The operating procedures for the devices consisted of 30 steps organized as four subprocedures. The steps within a subprocedure were classified as either formal or content steps. Content steps had an obvious relation to the function and operation of the device and were appropriate to the subprocedure in which they occurred. For example, one of the steps in the subprocedure for starting the engines on an airplane was to press an "Ignition" button. In contrast to content steps, formal steps had no obvious functional interpretation and could not be predicted from the nature of the device or subprocedure. For example, an operating procedure might entail pressing an "Enter" button or waiting for an "OK" light. However, the sequence of formal steps was always the same for each subprocedure in a given task. We refer to the sequence of formal steps followed in subprocedure as a procedural structure.

Two versions of each of two different types of devices were created. The two device types were an airplane control panel and an alarm system; the two different versions were created by using roughly synonymous labels for each of the steps and by altering the physical appearance of the device on the computer screen. Each type of device had its own sequence of subprocedures and appropriate content steps within each subprocedure, shown in Table 1. Two versions of two different procedural structures were also created, shown in Table 2. A total of eight experimental tasks were created by factorially combining the two device types (airplane and alarm), the two procedural structures (A and B), and the two versions ( 1 and 2 ) of the device and procedural structure.

Table 1

Devices, Subprocedures, and Content Steps in Experiment 1

\begin{tabular}{|c|c|c|c|}
\hline $\begin{array}{l}\text { Airplane } \\
\text { Version } 1\end{array}$ & $\begin{array}{l}\text { Airplane } \\
\text { Version } 2\end{array}$ & $\begin{array}{c}\text { Alarm } \\
\text { Version } 1\end{array}$ & $\begin{array}{c}\text { Alarm } \\
\text { Version } 2\end{array}$ \\
\hline \multicolumn{2}{|c|}{ Electrical Power } & \multicolumn{2}{|c|}{ Starting up } \\
\hline ain Power & Primary & Exterior & Outside \\
\hline Cabin Power & Cockpit & $\begin{array}{l}\text { Interior } \\
\text { Detectors }\end{array}$ & $\begin{array}{l}\text { Inside } \\
\text { Sensors }\end{array}$ \\
\hline
\end{tabular}

\begin{tabular}{ll}
\multicolumn{2}{c}{ Starting } \\
Ignition & Starter \\
Engine 1 & Propeller 1 \\
Engine 2 & Propeller 2
\end{tabular}

Communications Set up

Access Connect

Signal On Beacon

$$
\text { Communications Test }
$$

Start Send

Send Begin Test

Receive

\section{Configuring Sensors}

$\begin{array}{ll}\text { Motion } & \text { Movement } \\ \text { Continuity } & \text { Integrity }\end{array}$

\section{Testing System}

Answer Back Feedback
Control Response Alarm Signal Test

$\begin{array}{ll}\text { Timer On } & \text { Delay } \\ \text { Arm } & \text { On }\end{array}$

Table 2

Procedural Structures in Experiment 1

\begin{tabular}{ccccc}
\hline \multicolumn{2}{c}{ Structure A } & & \multicolumn{2}{c}{ Structure B } \\
\cline { 1 - 2 } \cline { 5 - 5 } Version 1 & Version 2 & & Version 1 & Version 2 \\
\cline { 1 - 2 } Select & Mode & & Select & Mode \\
Working & Processing & & [Content Steps] & [Content Steps] \\
Set & Input & & Working & Processing \\
[Content Steps] & [Content Steps] & Confirm & OK \\
Confirm & OK & & Set & Input \\
Enter & Done & & Enter & Done \\
\hline
\end{tabular}

Note-Steps on which subjects were to wait for a light to turn on are indicated in italics.

Design. There were four conditions determined by crossing structural similarity with conceptual similarity. In structurally similar conditions, the training and transfer tasks used the same procedural structure; in conceptually similar conditions, the training and transfer procedures used the same type of device (i.e., both were airplanes or both were alarms). Across subjects, each of the eight tasks was used equally often as a training and transfer task in each condition. However, subjects always learned different versions of the tasks during training and transfer

Procedure. Subjects first learned a practice task in order to become familiar with the mouse and the general features of operating the devices. The practice device was an answering machine with four buttons and two lights, and six steps were required in order to operate it. Following the practice task, subjects learned a training task and then a transfer task. Each task was learned to a criterion of two successful trials in a row. Whenever subjects made an error, a message appeared that informed subjects which button should have been pressed or which light they missed. Subjects could begin the next trial either at the beginning of the procedure or at the point of last error. If subjects elected to begin at the point of last error and reached the end of procedure without further errors, the trial was continued from the beginning of the procedure. Thus, each trial was terminated either by an error or by a successful completion of the entire procedure.

For each task, subjects were given two pages of instructions. The first page consisted of a general overview of the device and some of its functions. The second page consisted of an alphabetical listing of the buttons and lights along with a single-sentence, functional description of each. The subjects were not given any information about the sequence of steps that had to be performed, and they had to learn the procedures simply by trial-and-error. The subjects were instructed that they would have to operate the device two times in a row without error. They were allowed to keep the instruction booklet with them while they operated the device.

Subjects. The subjects were 64 volunteers who were paid for their participation. One subject in the conceptually similar/structurally dissimilar condition was replaced because he was unable to complete the training task in the amount of time allotted for the experimental session.

\section{Learning Model}

Our goal in the present research was not only to characterize which conditions produce more transfer than others but also to provide some account of how prior knowledge aids the acquisition of a new procedure. To that end, it is necessary to outline a framework for discussing and assessing the learning process. In our view, two general types of effects of prior knowledge on learning might be observed: Prior knowledge might provide a basis for performing a particular step in the procedure, or it might increase the speed with which a particular step 
is learned. In other words, prior knowledge may facilitate acquisition directly by applying immediately to the new task, or it may facilitate acquisition indirectly by increasing the learning rate. The notion of transfer of training suggests a direct effect on initial performance; however, it is also possible that indirect effects on learning rate may be observed. Thus, an adequate description of the role of prior knowledge requires that both kinds of effects be considered.

These two kinds of effects can be disentangled with a simple formalization of the learning process. For example, suppose that on each trial, a subject is either in a learned state or an unlearned state with respect to each step in the procedure, that the probability of being in the learned state initially is $s$, and that on each encounter with a particular step there is a fixed probability $r$ of moving from the unlearned state to the learned state. If subjects perform the step correctly when in the learned state and incorrectly when in the unlearned state, the expected number of errors would be

$$
N=(1-s) / r .
$$

In this formulation, $s$ reflects the immediately applicable knowledge that a user brings to a task, while $r$ reflects whatever knowledge, strategies, or processes are used in acquisition. This model is an all-or-none learning model and is referred to as the one-element model by Atkinson, Bower, and Crothers (1965).

Equation 1 can be used to characterize the effects of transfer. In particular, subjects may learn at a faster rate during the transfer task, and they may have learned some of the steps from their experience in the training task. If the total number of errors on a step on the training task is

$$
N_{1}=(1-s) / r_{1},
$$

the number of errors on the transfer task would be

$$
N_{2}=(1-s)(1-T) / r_{2},
$$

where $r_{2}$ is the learning rate on the second task and $T$ is the probability of transfer--that is, of knowing a step from previous experience of the training task. The number of parameters can be reduced by considering the relative error rate on the two tasks, $N_{2} / N_{1}$ :

$$
\frac{N_{2}}{N_{1}}=\frac{(1-T)}{R},
$$

where $R$ is the relative learning rate that is, $r_{2} / r_{1}$. In this description, the parameter $T$ reflects the direct effect of prior learning on performance, and $R$ reflects indirect effects on learning rate.

Although we have introduced Equations 1 and 2 in terms of an all-or-none learning model, they actually apply under a wide range of assumptions about the learning process. For example, if learning is characterized as incremental rather than all-or-none, one might assume that there is a probability $q_{i}$ of being correct on trial $i$, and that the probability increases by a constant proportion on each trial:

$$
q_{i+1}=q_{i}+\alpha\left(1-q_{i}\right) .
$$

This type of learning model has been referred to as a single-operator linear model (Bush \& Mosteller, 1955). In this model, the expected number of errors would be

$$
N=\left(1-q_{1}\right) / \alpha,
$$

which is formally identical to Equation 1 . The only substantive difference is the interpretation of the learning parameter: In Equation 1, $r$ is interpreted as the probability of learning a step on each encounter, while in Equation 3, $\alpha$ is interpreted as the increment in probability correct. The parameters $s$ and $q_{1}$ can both be interpreted as the probability of being correct on the first trial.

Results similar to Equations 1 and 2 are also obtained with a variety of other assumptions. For example, the random-trials increment model (Norman, 1964) is similar to the linear operator model, except that increments to $q$ do not occur on each trial but rather occur with some probability $c$. In effect, this model is a mixture of all-ornone learning and incremental learning. For example, if $c=1$, the model reduces to the single-operator model, and if the increment $\alpha=1$, the model is the same as the one-element model. Not surprisingly, the model makes comparable predictions for the total number of errors:

$$
N=\left(1-q_{1}\right) / c \alpha .
$$

In this case, $q_{1}$ reflects initial learning, while $c a$ characterizes the learning rate. In sum, Equation 1 can be used to describe the total number of errors in terms of initial knowledge and learning rate, regardless of whether learning is conceptualized as all-or-none, incremental, or somewhere in between.

Although these models make formally identical predictions for the total number of errors, they differ on a range of other predictions such as the variance in the number of errors, sequential statistics, and other aspects of the learning process. However, all of the models are consistent with an analysis of relative error rate based on Equation 2. Consequently, we do not need to decide among these models in order to use this formalization to assess the direct and indirect effects of transfer; Equation 2 can be viewed as a general property of a wide range of learning models that is independent of detailed assumptions of how learning proceeds. The approach adopted here is to use relative error rate as the principal dependent variable and to fit potential models of transfer to those results. Central to these analyses is the assumption that prior knowledge may have different effects on initial performance (i.e., $T$ ) and on rate of acquisition (i.e., $R$ ).

A variety of other approaches for characterizing transfer are also possible. For example, in principle, transfer could be assessed by simply evaluating performance on the transfer task: Performance should be better on the average in conditions with high transfer. However, our experience is that performance on tasks such as those used here is extremely variable across subjects, and the effects of transfer are unlikely to be observable against this background of variability. A common technique for dealing 
with subject variability in this type of design is to use an analysis of variance model with training/transfer task represented as a repeated measures factor. However, this approach implicitly assumes that subject variability and transfer effects are additive, and an examination of the data suggests that such an assumption would be inappropriate. For example, some subjects produced less than 10 errors on the initial training task, whereas other subjects made more than 50 . Our intuition is that transfer errors are unlikely to decrease by the same amount in instances such as these. In particular, a subject with a high error rate could easily improve by as many as 20 errors on the transfer task, and it would be impossible for a subject with an initially low number of errors to improve by that much. Instead, it seems much more reasonable to expect that the reduction on the transfer task would be proportional in some way to performance on the training task; in other words, we assume that subjects who make a large number of errors during training have more room to improve and should show proportionately larger effects of transfer. Thus, in our view, using relative error rate $\left(N_{2} / N_{1}\right)$ to measure transfer is intuitively reasonable in addition to being theoretically justified.

Relative error rate is not the only possible measure of proportional improvement. For example, one could consider the reduction in errors relative to the total number of errors $\left(N_{2}-N_{1}\right) /\left(N_{2}+N_{1}\right)$, or the number of errors on the transfer task relative to the total number of errors $N_{2} /$ $\left(N_{2}+N_{1}\right)$. However, such measures are monotonic transformations of the relative error rate and produce similar results and interpretations. A further analytical issue concerns the measure of task performance. Instead of the total number of errors, one could index performance by using variables such as trial of last error or total number of trials. However, in our data, these measures are highly correlated with the number of errors. In addition, these measures do not lend themselves to a simple formulation (such as Equation 1) that is independent of specific assumptions about the nature of learning. In sum, we used relative error rate as a measure of transfer in this work because it provides a simple, intuitive measure of transfer and because it allows a parametric decomposition of direct and indirect transfer effects that does not depend on specific assumptions of the learning model.

\section{Results}

Step-specific transfer. Relative error rate was calculated for each subject, and the mean values are shown in Figure 1; for completeness, the total number of errors for each task is shown in Table 3 . Figure 1 also shows the predictions of the step-specific transfer hypothesis as straight lines. These predictions were generated by assuming that conceptual similarity would lead to transfer on content steps and that structural similarity would lead to transfer on formal steps; this transfer would be reflected in nonzero values of the parameter $T$ for content and formal steps in those conditions. Further, it was assumed that the relative learning rate, $R$, was potentially different for content and formal steps but was constant across condi-

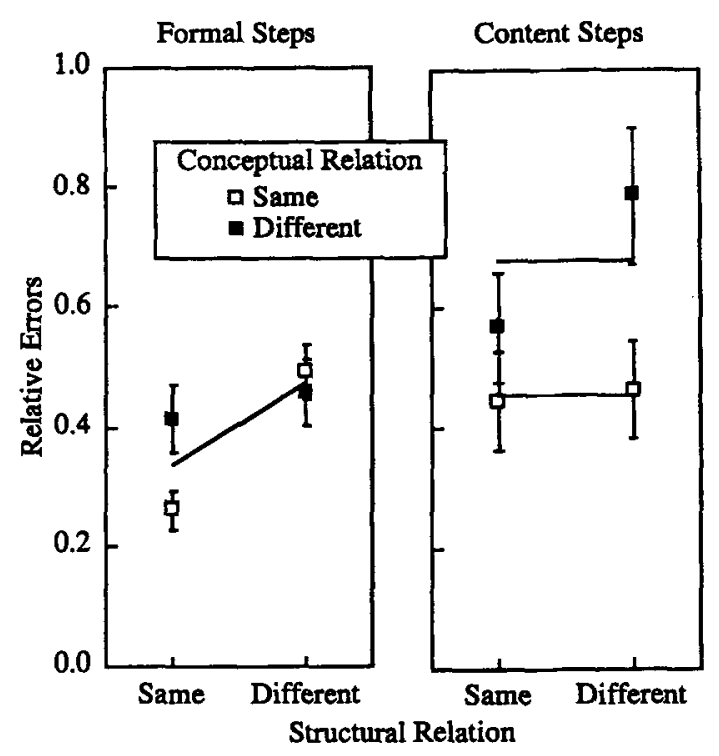

Figure 1. Fit of the step-specific transfer hypothesis to the relative error rates in Experiment 1.

tions. In effect, these assumptions imply that there should be a main effect of conceptual similarity on content steps and a main effect of structural similarity on formal steps. Four parameters were estimated in order to fit the data: a value of $T$ on content steps in conceptually similar conditions, a value of $T$ on formal steps in structurally similar conditions, a value of $R$ for content steps generally, and a value of $R$ for formal steps generally. Least-squares estimates of these parameters are shown in Table 4.

Figure 1 makes it clear that although the hypothesis of step-specific transfer accounts for some of the trends in the data, there are consistent deviations from the predictions. On the positive side, the hypothesis correctly predicts that formal steps are generally easier when the devices are structurally similar and that content steps are generally easier when the devices are conceptually similar. However, there are substantial interactions between conceptual and structural similarity that are not predicted by step-specific transfer. In particular, there is a sizable advantage on formal steps for tasks that are both conceptually and structurally similar, and structural similarity has an effect on content steps even in the absence of conceptual similarity. These differences suggest that the simple hypothesis of step-specific transfer is inadequate.

Routine-specific transfer. A variety of hypotheses can be generated for the relatively poor fit of the step-specific transfer model. However, our intuition was that one of the model's major shortcomings is that it assumes no role for information about the overall organization of the procedures, even though the decomposition of the procedures into subprocedures was a salient characteristic of the experimental task. Consequently, we developed an alternative formulation based on the idea that subjects explicitly generate representations of the task subprocedures. Such an approach is consistent with evidence that indicates 
Table 3

Mean Total Errors (and Standard Errors) in Experiment 1

\begin{tabular}{|c|c|c|c|c|c|c|c|c|}
\hline & \multicolumn{4}{|c|}{ Training Task } & \multicolumn{4}{|c|}{ Transfer Task } \\
\hline & \multicolumn{2}{|c|}{$\begin{array}{l}\text { Formal } \\
\text { Steps }\end{array}$} & \multicolumn{2}{|c|}{$\begin{array}{c}\text { Content } \\
\text { Steps } \\
\end{array}$} & \multicolumn{2}{|c|}{$\begin{array}{l}\text { Formal } \\
\text { Steps }\end{array}$} & \multicolumn{2}{|c|}{$\begin{array}{c}\text { Content } \\
\text { Steps }\end{array}$} \\
\hline & $M$ & $S E$ & $M$ & $S E$ & $M$ & $S E$ & $M$ & $S E$ \\
\hline $\begin{array}{l}\text { Conceptually similar/ } \\
\text { structurally similar }\end{array}$ & 15.2 & 1.9 & 11.8 & 1.8 & 4.4 & 1.2 & 4.9 & 1.2 \\
\hline $\begin{array}{l}\text { Conceptually similar/ } \\
\text { structurally dissimilar }\end{array}$ & 12.1 & 1.9 & 9.4 & 1.5 & 6.1 & 1.1 & 4.4 & 1.0 \\
\hline $\begin{array}{l}\text { Conceptually dissimilar/ } \\
\text { structurally similar }\end{array}$ & 16.3 & 2.7 & 11.6 & 1.4 & 6.4 & 1.1 & 5.6 & 0.6 \\
\hline $\begin{array}{l}\text { Conceptually dissimilar/ } \\
\text { structurally dissimilar }\end{array}$ & 17.5 & 2.1 & 9.4 & 1.2 & 7.6 & 1.1 & 6.1 & 0.7 \\
\hline
\end{tabular}

that when solving complex problems, subjects segment their problem solution into sequences of steps that accomplish particular subgoals (e.g., Newell \& Simon, 1972). In the present context, we assume that subjects generate a representation of the exact sequence of steps needed to carry out some portion of the procedure (typically a subprocedure). We refer to such a representation as a routine. Our hypothesis is that routines are the principle source of transfer effects in the present task.

The concept of routines can be used to explain the pattern of results in Experiment 1 in the following way. When subjects encounter a familiar device as the transfer task (e.g., a different alarm system after having learned an alarm as the training task), subjects hypothesize that the device is operated in a similar fashion and retrieve routines learned during the training task. Transfer should be observed on content steps because these steps match those in the retrieved routines; subjects merely have to identify the step in a given routine with the corresponding (synonymous) step in the transfer task. If the transfer task is also structurally similar to the training task, transfer should also be observed for formal steps because these steps will match as well. However, in structurally dissimilar conditions, the formal steps would be entirely different from those learned during training; in this case, the formal steps would have to be learned through trial and error. However, neither content steps nor formal steps would benefit in the absence of conceptual similarity because there would be no basis for retrieving routines learned from the training task. In sum, we hypothesize that conceptual and structural knowledge is stored together (as routines) and that routines are retrieved on the basis of conceptual cues. Thus, formal steps benefit only when tasks are both conceptually and structurally similar, but content steps benefit from conceptual similarity regardless of the structural relation.

One further assumption is required in order to provide an accurate account of the experimental results. An examination of Figure 1 indicates that structural similarity has an effect on relative errors, even in the absence of conceptual similarity; such a result would not be expected if transfer was mediated solely by the retrieval of routines. We hypothesize that this effect occurs because structural similarity improves the learning rate. On this hypothe- sis, structural similarity allows subjects to segment the procedure and leads them to try certain kinds of steps at certain points in the procedure. For example, after learning a task using procedural structure A, subjects may expect to start each subprocedure by the same sequence of steps: pressing a button, waiting for a light, and then pressing another button. And, in learning the new procedure, subjects may search for those kinds of steps first before looking for more meaningful content steps. This strategy would produce an advantage when the devices are structurally similar: The range of possible steps that would have to be considered at any point in the task would be restricted, and subjects would be able to find the correct step more quickly.

Figure 2 shows the fit of the routine-specific transfer hypothesis to the relative error rates in Experiment 1. The fit required four parameters. As with step-specific transfer, $R$ was assumed to be different for formal and content steps. The advantage in learning rate for structural similarity was modeled by multiplying $R$ by a further factor, $w$, in those conditions. Conceptual similarity was assumed to lead to a nonzero value of $T$ for content steps and for similar formal steps. Least-squares parameter estimates are shown in Table 5. According to the estimated parameters, formal steps were learned about twice as fast during transfer as during training, and content steps were learned about $30 \%$ faster. The estimated value of $T$ indicates that when prior knowledge could be applied to the transfer task, there was about a $30 \%$ chance of already knowing a particular step; this improvement occurred on content steps when the training and transfer tasks were conceptually similar and on formal steps when the tasks were both conceptually and structurally similar. The estimated value of $w$ indicates that structural similarity by itself led to an improvement of about $20 \%$ in learning rate.

As is clear from the figure, the fit to the data is essentially perfect: The model accounts for $96 \%$ of the variance across all of the conditions, and each data point is predicted to within half a standard error. There is no consistent departure from the predictions, and the remaining deviations can be plausibly attributed to experimental error. Further, unlike the fit obtained on the hypothesis of step-specific transfer, the model predicts all of the substantial effects found in the data, and the qualitative pattern of predictions is virtually identical to the obtained results (e.g., the rank-order correlation between data and predictions is .98). Despite the post hoc nature of the model, we regard this fit as compelling evidence for the adequacy of the model.

Table 4

Parameter Estimates for the Step-Specific Transfer Hypothesis in Experiment 1

\begin{tabular}{cc} 
Transfer Hypothesis in Experiment 1 \\
\hline Parameter & Value \\
\hline$R$ (formal steps) & 2.11 \\
$R$ (content steps) & 1.47 \\
$T$ (formal steps) & 0.29 \\
$T$ (content steps) & 0.33 \\
\hline
\end{tabular}




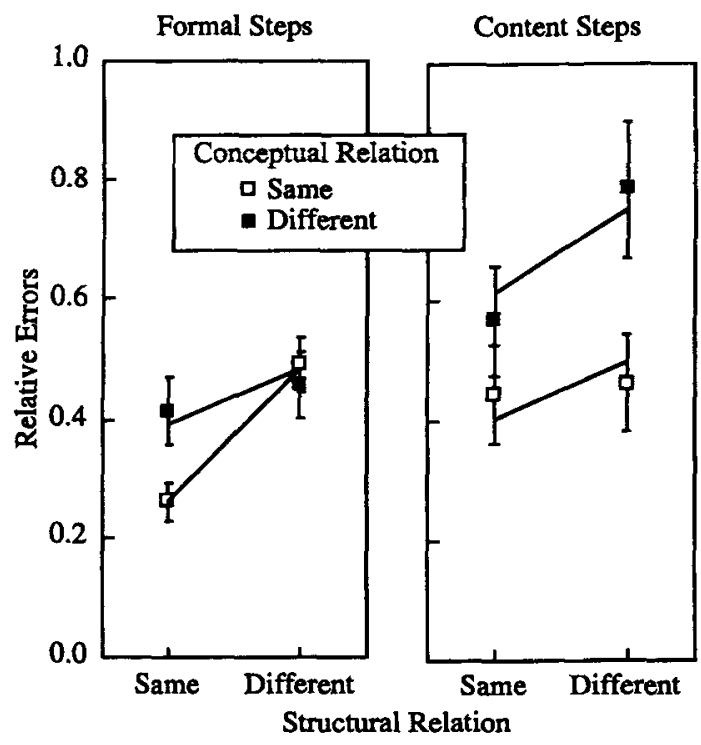

Figure 2. Fit of the routine-specific transfer hypothesis to the relative error rates in Experiment 1.

It might be suggested that an accurate fit to the results is inevitable given that four parameters were estimated in order to fit eight data points. However, four parameters is arguably the smallest number of parameters one would need in order to fit any model to results from a design of this sort. By way of comparison, consider what would be involved in applying the linear model used in analysis of variance. The simplest possible pattern of results that one could expect might involve a single main effect on contentstep transfer and a single main effect on formal-step transfer. An analysis of variance model would thus require four parameters: one for each of the two main effects, one for the overall mean on content steps, and one for the overall mean on formal steps. Thus, the routine-specific model of transfer is no more elaborate than an exceedingly simple linear model. Of course, what is different about the routine-specific model relative to a typical analysis of variance model is that it is based on a theoretically guided analysis of how prior knowledge affects acquisition rather than being assumed by default. In any event, the important point to be taken from these results is that the hypothesis of routine-specific transfer makes much more accurate predictions than the hypothesis of stepspecific transfer while requiring no more parameters.

\section{Discussion}

Although the results support the importance of distinguishing conceptual and structural knowledge, they are inconsistent with the simple notion of step-specific transfer. That is, the value of conceptual similarity is not restricted to content steps, and the value of structural similarity is not restricted to formal steps. Consequently, we developed an alternative interpretation of the results, based on the idea that transfer is mediated primarily by retrieval of routines from memory. On this new account, both content and formal steps may benefit when an ap- propriate routine is found. Conceptual similarity has a special role in this formulation, however, because it provides effective cues for searching memory for relevant routines. This use of conceptual similarity is reminiscent of Ross's $(1984,1987,1989)$ results on remindings in problem solving. In effect, we suspect that the use of similar devices functions as a reminding that aids in the finding of appropriate routines.

Our conclusion that transfer is mediated primarily by memory retrieval is different from that reached by Kieras and Bovair (1984). They argued that conceptual information improves performance because it aids reasoning and inference processes, not memory per se. Of course, the situation examined here is quite different from that examined by Kieras and Bovair. Subjects in those experiments were asked to perform the task without any prior experience of similar procedures and were also given a wealth of detailed information about the operation of the device. In contrast, the present task was set up explicitly to look at effects of prior experience, and the information about the operation of the device was relatively impoverished. Thus, it is not surprising that different conclusions are suggested by the two studies. However, our guess is that the present experimental situation is more likely to be of practical significance; that is, we suspect that similarity to previously learned procedures may guide a large proportion of the procedural learning that occurs in the world.

In the model of routine-specific transfer, we also assumed that there was a separate effect of structural similarity, independent of retrieving routines from memory. Our view is that structural similarity is likely to aid in the process of decomposing tasks into subprocedures. This hypothesis is consistent with the results reported by Dixon and Gabrys (1991) and Kamouri et al. (1986). In Dixon and Gabrys, for example, structurally similar devices used precisely the same organization of procedures into subprocedures, and many of subprocedures involved controls (such as a numeric keypad) that were similar across training and transfer. Thus, structural similarity could easily have provided the basis for accurate task decomposition. In Kamouri et al., conceptually different, structurally similar devices used identical sequences of button presses to control device states. Such common sequences would provide a clear indication of the appropriate segmentation of the procedure. Thus, we suspect that the major benefit of structural similarity, in both the present experiment and these earlier ones, was not to provide specific information about particular steps, but rather to aid learning by providing a readily accessible task decomposition.

Table 5

Parameter Estimates for the Routine-Specific Transfer Hypothesis in Experiment 1

$\begin{array}{ll}\text { Parameter } & \text { Value } \\ R \text { (formal steps) } & 2.07 \\ R \text { (content steps) } & 1.33 \\ T & 0.34 \\ w & 1.23\end{array}$




\section{EXPERIMENT 2}

Experiment 2 was designed to provide a further test of our interpretation of the results of Experiment 1 . The hypothesis advanced here is that subjects retrieve routines for the task subprocedures. Further, we assume that each routine consists of an unanalyzed list of the steps to be performed. This view entails that subjects must understand enough about the nature of a task to generate relevant retrieval cues, but that once a routine has been successfully retrieved, the steps can be performed with little effort or reflection. In particular, we suppose that subjects generally do not make inferences about what steps would be required in order to carry out a particular subprocedure. This view was tested in Experiment 2 by rearranging the order of steps within each subprocedure from training to transfer. Because routines contain only a list of the steps to be performed, the retrieved routines would be of little help if the subprocedure requires the steps to be performed in a different order. Thus, the hypothesis of routine-specific transfer predicts that there should be no basis for transfer unless the order of steps within subprocedures is precisely the same from training to transfer.

These predictions of routine-specific transfer are not inevitable, and there are alternative interpretations of the results of Experiment 1 that generate entirely different predictions. In particular, research on problem solving suggests a contrasting analysis. When people solve complex problems, it is common to generate subgoals for achieving major aspects of the task and to attempt to solve each of the subgoals as separate problems (see, e.g., Newell \& Simon, 1972). For example, when attempting to calculate the probability of an event in a probability problem, subjects may generate the subgoal of first finding the overall frequency of the event and then after that subgoal has been achieved, divide by the total number of trials to solve the original problem (Catrambone \& Holyoak, 1990). Catrambone $(1994,1995)$ has argued that the appropriate use of subgoals in problems like this promotes transfer: Learners are able to generalize to novel tasks more readily because they can find new solution methods to achieve the same sequence of subgoals.

Transfer in the present task may be mediated by a similar process. For example, during the training task, subjects may generate subgoals corresponding to each of the subprocedures and then attempt to find steps that would achieve each of those subgoals in turn. Then, during the transfer task, subjects would retrieve the information about the subgoals that were used during training and search for new steps that would achieve those same subgoals in the transfer task. In the present case, we assume that subjects would have learned the sequence of subgoals used in the training task and that they would attempt to use this sequence in performing the transfer task. On this analysis, conceptual similarity improved transfer in Experiment $l$ because it allowed subjects to retrieve the correct sequence of subgoals, which in turn allowed them to identify the required steps more readily.
If transfer in this task is mediated by knowledge of the subgoal sequence in this way, rearranging the order of steps within a subprocedure should not eliminate transfer. As long as the order of the subprocedures themselves remains intact, subjects would know the appropriate subgoal at each point in the procedure. This knowledge in turn should allow subjects to make plausible guesses about which steps are required, even if the order of those steps is different from that learned during training. Of course, if precisely the same sequence of steps is used to achieve a subgoal during transfer as during training, subjects should do quite well in the transfer task. However, the crucial prediction for this view is that subjects should be able to make inferences about what kinds of steps should be performed even when the steps have been rearranged within a subprocedure, and at least some transfer should be observed under these conditions.

Routine-specific transfer and subgoal-specific transfer also make different predictions for the effect of rearranging the subprocedures themselves. If routines are retrieved from memory, varying the order of the subprocedures should have little effect on transfer: As soon as subjects can identify the nature of the task, they should be able to retrieve appropriate routines and apply them to the new task with little difficulty. In contrast, if transfer is based on a learned sequence of subgoals, rearranging the order of subprocedures should produce a substantial reduction in transfer. That is, the subgoal sequence learned from the training task would not be sufficient to identify relevant subgoals in the new task, and consequently it would be more difficult to identify the appropriate content steps. In sum, according to the hypothesis of routine-specific transfer, transfer should depend critically on a consistent order of steps within each subprocedure; according to subgoal-specific transfer, transfer should depend on a consistent order of subprocedures from training to transfer. These contrasting predictions were tested in Experiment 2.

\section{Method}

Apparatus and Procedure. The apparatus and procedure was identical to those in Experiment 1. The only difference lay in the nature of the materials and the relations between training and transfer tasks.

Materials. Two versions of the airplane device were used in Experiment 2. The tasks were similar to those used in Experiment 1, although some of the subprocedures and content steps were modified to eliminate temporal-order constraints. All of the tasks used procedural structure A from Table 2. As is shown in Table 6, there were two possible orders of the content steps in the subprocedures. In addition, there were two possible orders for the subprocedures. The first order is shown in Table 6: Flight Contrc!s, Cabin Power, Communications Setup, and Engine Start-Up. The second order was Communications Setup, Flight Controls, Engine Start-Up, and Cabin Power. In total, there were eight possible tasks, consisting of the two versions of the device, two possible subprocedure orders, and two possible step orders within subprocedures.

Design. Two factors were manipulated in this experiment: The order of the subprocedures could be the same or different from the training task to the transfer task, and the order of the steps within each subprocedure could be the same or different. There were four between-subjects conditions generated by the factorial combination 
Table 6

Content-Step Orders in Experiment 2

\begin{tabular}{|c|c|c|c|}
\hline \multicolumn{2}{|c|}{ Step Order A } & \multicolumn{2}{|c|}{ Step Order B } \\
\hline Version 1 & Version 2 & Version 1 & Version 2 \\
\hline \multicolumn{4}{|c|}{ Flight Controls } \\
\hline Rudder & Tail Controls & Flaps & Wing Controls \\
\hline Flaps & Wing Controls & Rudder & Tail Controls \\
\hline \multicolumn{4}{|c|}{ Cabin Power } \\
\hline Overhead Lights & Cabin Illumination & Warning Lights & Emergency Lights \\
\hline Ventilation & & Overhead Lights & Cabin Illumination \\
\hline Warning Lights & Emergency Lights & Ventilation & Air \\
\hline \multicolumn{4}{|c|}{ Communications Set up } \\
\hline Access & Connect & Signal On & Beacon \\
\hline Signal On & Beacon & Access & Connect \\
\hline \multicolumn{4}{|c|}{ Engine Start up } \\
\hline Engine 1 & Propeller 1 & Ignition & Starter \\
\hline Engine 2 & Propeller 2 & Engine 1 & Propeller 1 \\
\hline Ignition & Starter & Engine 2 & Propeller 2 \\
\hline
\end{tabular}

of these two factors. As before, each of the eight tasks was used equally often as a training task and as a transfer task across subjects. If the first device version was used during training, the second version was used during transfer and vice versa.

Subjects. The subjects were 65 undergraduate psychology students who participated for course credit. Data from one of these subjects were not used because of error by an experimenter during the session.

\section{Results}

Routine-specific transfer. The relative error rates are shown in Figure 3 along with the fit of the RoutineSpecific Transfer model; for completeness, the mean number of errors in each condition on the training and transfer tasks is shown in Table 7. Two values of $R$ and one value of $T$ were estimated to generate the fit in Figure 3. As in Experiment 1, separate values of the relative learning rate, $R$, were estimated for content and formal steps. $T$ was assumed to be zero for content steps when the order of steps within a subprocedure was misordered, and a common value of $T$ was used for the other conditions. The estimated parameter values are shown in Table 8 . The estimated parameters suggest that formal steps were learned about $60 \%$ faster during transfer as during training, whereas content steps were learned about $10 \%$ more slowly. The estimated value of $T$ indicates that when the same order of steps was used within subprocedures, there was a $40 \%$ chance of knowing a step from experience in the training task.

As is shown in Figure 3, the model fits the obtained results with some precision, accounting for $98 \%$ of the variance across conditions. The predicted values were virtually identical to the obtained values for all but two of the data points, and even for these the prediction error was substantially less than a standard error. There is little variation in formal steps in any of the conditions because these steps were always similar across the training and transfer tasks. For content steps, the model predicts that relative error rate should increase when the order of steps within a subprocedure is misordered and that there should be little effect of the order of subprocedures. This prediction is reasonably close to the obtained results, although there is a suggestion that the error rate may decline somewhat when the transfer task matches the training task at both the subprocedure and step levels.

Subgoal-specific transfer. Figure 4 shows the fit generated by the hypothesis that transfer is mediated by the sequence of subgoals. Clearly, the hypothesis does not capture important trends in the results for content steps (Figure 4, right panel). Consider, first, the pattern of results when the subprocedure order is unchanged from training to transfer (i.e., the left points in the right-hand panel). Here, the hypothesis successfully accounts for the difference between same and different step order (i.e., the difference between the filled point and unfilled point). The error rate is predicted to be lower when the content steps are exactly the same at training and transfer because subjects are expected to remember at least some of the steps. Now consider the results when subprocedures are reordered from training to transfer (i.e., the rightmost points in Figure 4). In this case, the hypothesis fails to predict any effect of step order within a subprocedure. In both of these conditions, the content steps are not exactly the same from training to transfer and so, by hypothesis, must be relearned. The hypothesis of subgoal-specific transfer provides no account of the substantial effect of step order obtained in this case. Finally, consider the re-

Table 7

Mean Total Errors (and Standard Errors) in Experiment 2

\begin{tabular}{|c|c|c|c|}
\hline \multicolumn{2}{|c|}{ Training Task } & \multicolumn{2}{|c|}{ Transfer Task } \\
\hline $\begin{array}{l}\text { Formal } \\
\text { Steps }\end{array}$ & $\begin{array}{l}\text { Content } \\
\text { Steps }\end{array}$ & $\begin{array}{l}\text { Formal } \\
\text { Steps }\end{array}$ & $\begin{array}{l}\text { Content } \\
\text { Steps }\end{array}$ \\
\hline
\end{tabular}

Same subprocedure order/ same step order

Same subprocedure order/ different step order $\begin{array}{llllllll}10.4 & 1.2 & 6.7 & 0.6 & 3.6 & 0.6 & 3.8 & 0.6\end{array}$

Different subprocedure order/ same step order $\begin{array}{llllllll}9.3 & 0.9 & 6.1 & 0.7 & 3.1 & 0.5 & 5.6 & 0.6\end{array}$ Different subprocedure order different step order 
Table 8

Parameter Estimates for the Routine-Specific Transfer Hypothesis in Experiment 2

\begin{tabular}{cc}
\hline Parameter & Value \\
\hline$R$ (formal steps) & 1.57 \\
$R$ (content steps) & 0.89 \\
$T$ & 0.40 \\
\hline
\end{tabular}

sults when the step order within subprocedures was different at training and transfer (i.e., the filled points in the right-hand panel). Here, the hypothesis predicts in principle that relearning should be easier when the same sequence of subgoals is used. However, this effect was not obtained; instead, the error rate in these conditions was roughly equivalent (i.e., there is no difference between the two filled points).

The fit shown in Figure 4 required four parameters. As before, $R$ was assumed to be different for content and formal steps. A common value of the transfer parameter, $T$, was estimated for formal steps, as well as for content steps when both the subprocedure order was the same and the step order within a subprocedure was the same; $T$ was assumed to be zero for content steps in the other conditions. However, according to the hypothesis, when the order of subprocedures is the same, subjects should be able to learn the appropriate steps more quickly even when precise knowledge of the order of steps is unavailable. This prediction was modeled by multiplying the learning rate by a factor, $w$, in those conditions; $w$ was constrained to be greater than or equal to 1 . The estimated parameter values are shown in Table 9.

\section{Discussion}

The results suggest that the most important aspect of the memory representation of a previously learned procedure is the order of the steps within a subprocedure. Presumably, this reflects the organization of prior experience into routines that specify sequences of steps needed in order for one to perform particular subtasks. Further, the results suggest that prior experience of a subprocedure does not improve transfer when a new sequence of steps is required in order to accomplish that subtask, despite the fact that the general nature of the steps could be predicted from the meaning of the subprocedure. For example, suppose that during training a subject learns that the first subprocedure involves the flight control "Rudder," followed by "Flaps." During transfer, that subject should be able to predict that similar flight controls should be involved in the first subprocedure (e.g., "Wing Controls" and "Tail Controls"); given this prediction, there would be only a small number of candidate buttons to try at each step. Thus, one might expect a relatively low error rate when the subprocedure order was the same, even if the corresponding steps were in a different order. However, this result was not obtained, suggesting that subjects did not use this kind of prediction strategy. Instead, transfer was found only when the intact sequence of steps to accomplish a particular subprocedure could be retrieved from memory (e.g., as a routine).
A second important aspect of the results is that the order of subprocedures had relatively little effect on transfer. For example, when the same step order was used during training and transfer, there was little difference between conditions in which the same subprocedure order was used and those in which a different subprocedure order was used (i.e., there is little difference between unfilled points in Figure 4). This suggests that subjects do not use information about the order of routines needed to carry out the task as a whole. If the order of subprocedures was more salient to subjects, one might have expected a larger difference between these conditions. Note that in order to perform the task correctly during training, subjects must learn the order of the subprocedures. However, the failure to find a large effect of subprocedure order on relative error rate during transfer suggests that this information is not reliably retrieved and applied to the transfer task.

This result raises an interesting question about how subjects performed the transfer task: If subjects did not make use of the previously learned subprocedure order in learning the new task, how could subjects decide which of the retrieved routines should be used at each point in the task? One possibility is that subjects apply routines by trial and error. At the beginning of each subprocedure, they could select one of the routines learned in the training task and attempt to apply it to accomplish the current subprocedure. If the routine produced an error, they would select a different routine from memory and try to apply that to the new task. This process would continue until the appropriate routine was found. Because there were only four subprocedures (and only four routines) involved in the tasks used here, this approach would have worked moderately well in some conditions. In particular, it would have produced a minimum number of errors as long as the order of the steps within each subprocedure remained the same from training to transfer. However, if

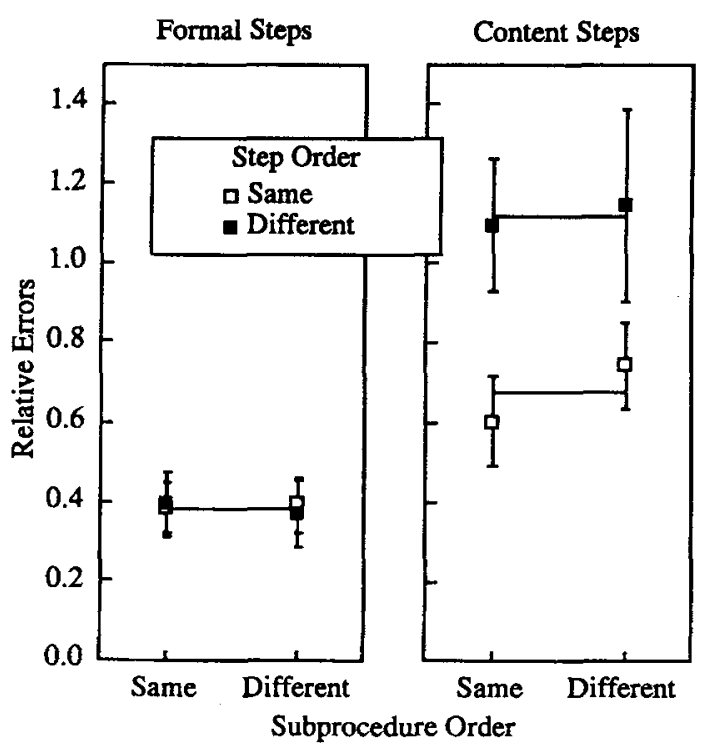

Figure 3. Fit of the routine-specific transfer hypothesis to the relative error rates in Experiment 2. 


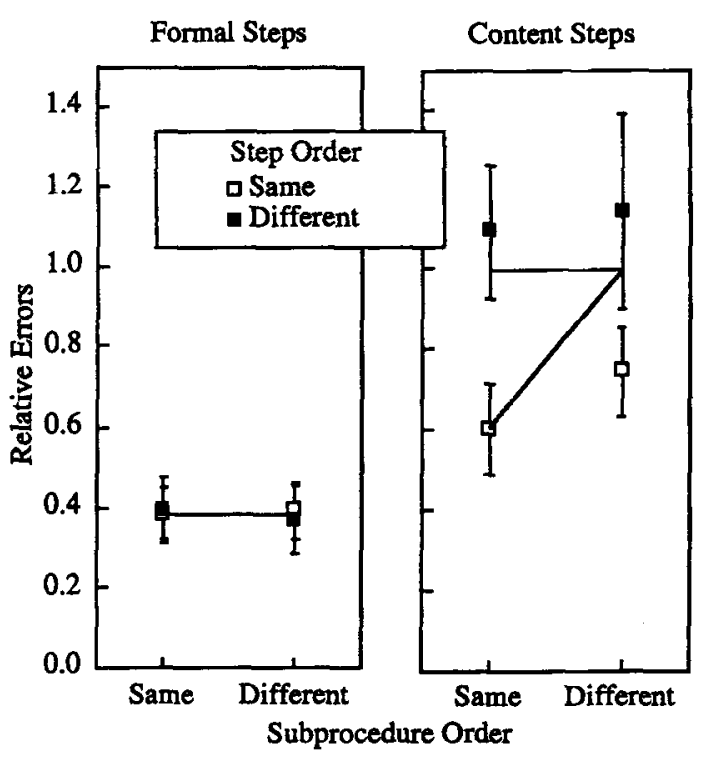

Figure 4. Fit of the subgoal-specific transfer hypothesis to the relative error rates in Experiment 2.

the steps were rearranged within a subprocedure, this strategy would have produced poor performance, because none of the routines learned during training would work. This prediction is consistent with the obtained results.

The results of Experiment 2 also provide an explanation for a discrepancy between the results of Dixon and Gabrys (1991) and those obtained in Experiment 1. In the earlier study, conceptual similarity between training and transfer had no appreciable effect on performance; however, substantial effects of conceptual similarity on both formal and content steps were observed in Experiment 1. A close examination of the manipulation of conceptual similarity accounts for this difference. In the earlier experiments of Dixon and Gabrys, conceptually similar devices were similar only at the level of subprocedures. In particular, the content steps in the subprocedures were always arranged differently from training to transfer. Thus, the manipulation of conceptual similarity was analogous to the same-subprocedure-order/different-step-order condition of Experiment 2 . In this condition, relatively little effect of conceptual similarity was observed, just as was found by Dixon and Gabrys. In both of the experiments reported here, substantial effects of conceptual similarity were observed only when the sequence of steps was precisely the same at training and transfer, unlike the conditions used in the earlier study.

Table 9

Parameter Estimates for the Subgoal-Specific Transfer Hypothesis in Experiment 2

\begin{tabular}{cc}
\multicolumn{2}{c}{ Transfer Hypothesis in Experiment 2} \\
\hline Parameter & Value \\
\hline$R$ (formal steps) & 1.58 \\
$R$ (content steps) & 1.00 \\
$T$ & 0.39 \\
$w$ & 1.00 \\
\hline
\end{tabular}

The manipulation of subprocedure order is critical with respect to only one version of how subgoals might be used in the present task. In particular, we assumed that if subjects learn a sequence of subgoals during training, that sequence would be unusable when the transfer task used a different sequence of subprocedures. However, it is possible that subgoals for performing the task might be acquired during training without necessarily being organized as a strict sequence. On this view, one might expect to see some benefit of subgoal learning regardless of whether subprocedures were reordered or not. One problem for this proposal is that it is not clear how subjects decide which subgoal is relevant at each point in the task. In any event, the results shown in Figures 3 and 4 do not provide support for this interpretation: When steps are reordered within a subprocedure, the relative error rate on content steps was close to 1 , implying that there was little transfer under those conditions. If subjects can use learned subgoals to infer possible steps in a subprocedure, one would expect to have seen at least some transfer in all conditions.

\section{GENERAL DISCUSSION}

The original goal of this research was to relate the distinction between conceptual knowledge and structural knowledge to the acquisition of content steps and formal steps in a procedure. In our terminology, conceptual knowledge concerns how a device functions or why a procedure works; as such, it is embedded in a field of more general semantic knowledge about the world. On the other hand, structural knowledge concerns the structure and organization of the procedure itself. Although structural knowledge might generalize to similar devices or procedures, it has little semantic content and consequently cannot participate in more general causal inferences. We hypothesized that the steps in a procedure that could be predicted on the basis of conceptual knowledge (content steps) would show a benefit of previous experience with conceptually similar devices, whereas the steps that were involved in the organization of the procedure (formal steps) would show a benefit of previous experience with structurally similar devices. This pattern of results failed to occur; instead, the results displayed a complex interaction in which both procedural and structural knowledge affected both content and formal steps.

Our interpretation of these results is that transfer in tasks such as ours is not mediated solely by reasoning or problem solving. Instead, memory retrieval plays a central role. We hypothesize that during acquisition of a procedure, users learn sequences of steps that accomplish task subprocedures and that such sequences include both content steps and formal steps. We refer to the memory representations of such sequences as routines. Our view is that routines are independent of one another and are relatively uninterpreted. In particular, users may have little insight into why certain steps are required in a subprocedure and not others. We hypothesize that routines 
are retrieved on the basis of superficial similarity between tasks: When a new task seems similar to a previously learned task, the user retrieves the routines that were used in the earlier task and attempts to apply them in the new context. We suspect that this process typically does not depend on deep conceptual analysis of the task or device. Instead, the routines may be applied to the new task in a haphazard or random fashion, using trail and error. According to this view of procedural transfer, the major task facing the learner of a new task is not causal analysis or problem solving, but rather retrieval of known routines that are relevant in the current context.

This general conception explains many aspects of the present results. In Experiment 1, substantial transfer was observed only when the procedures were conceptually similar. Presumably, conceptual similarity provided the retrieval cues that were needed for one to find the relevant routines learned during training; without this apparent similarity, subjects did not attempt to apply their previously learned routines. In Experiment 2, transfer on content steps was observed only when the sequence of steps within a subprocedure was intact; in other words, acquisition of content steps benefited from the retrieved routines only when the required step sequence precisely matched that in routine. However, transfer was observed on formal steps in all conditions, presumably because these steps always matched the corresponding steps in the retrieved routine. Moreover, in Experiment 2, there was relatively little effect of reordering the subprocedures from training to transfer. This supports the view that the routines are unordered and independent of one another.

We suspect that the effects of structural similarity observed in Experiment 1 are due to a somewhat different mechanism. Structural similarity may aid the user in isolating segments of the task that correspond to different subprocedures. The ability to parse a task in this way may improve learning when users fail to find the correct routine; in particular, it would limit the kinds of steps that need to be considered at each step of the procedure. For example, in the present context, structural similarity would allow the user to infer when to perform a content step and when to perform a formal step, or when to wait for a light or to search for a button. In other words, our hypothesis is that structural similarity comes into play when retrieval fails, by helping the user to decompose the task into more manageable components. However, the present results provide little insight into the nature of the structural knowledge, how it is organized, or how it is used.

Several other accounts of transfer with complex procedures failed to provide adequate characterizations of the present results. For example, a priori it seemed reasonable to suspect that transfer would be specific to the nature of the steps. In particular, we expected that conceptual similarity would improve performance on content steps and that structural similarity would improve performance on formal steps. These results did not occur; the hypothesis of step-specific transfer failed to account for effects of conceptual similarity on formal steps or for the effects of structural similarity on content steps. Similarly, the results of Experiment 2 suggest that the effect of conceptual similarity cannot be explained by the learning of sequences of subgoals. On such a view, one would expect the manipulation of subprocedure order to have large effects on performance and the manipulation of step order to have small effects, and this was demonstrably not the case. In the balance, we believe that the hypothesis of routine-specific transfer to be the most accurate and parsimonious account of these results.

In conclusion, our account of these results suggests an interesting conjecture regarding the role of conceptual knowledge in the learning of complex procedures generally. We hypothesize that under a wide range of circumstances, the benefit of elaborate and detailed conceptual knowledge in a domain may be to aid in the retrieval of previously learned routines. In particular, users may rarely use their conceptual knowledge to reason about the operation of a device or the function of a procedure. Instead, they may use their knowledge in a more or less automatic way to find sequences of steps that they already know how to perform, rather than attempt to infer logically what those steps must be. Although this hypothesis is consistent with the view that conceptual knowledge aids in the acquisition of new procedures, it suggests a different emphasis than that which has been common in previous research. For example, it may be the case that even when users have a detailed conceptual knowledge, the relationship between the precise sequence of steps needed to perform a novel task and those used in performing similar tasks is crucial in determining how easily the new procedure will be acquired. In general, we conjecture that memory retrieval is the crucial ingredient in how users acquire new procedures, and that reasoning and problem solving processes may not be as important as one might suspect.

\section{REFERENCES}

Atkinson, R. C., Bower, G. H., \& Crothers, E. J. (1965). An introduction to mathematical learning theory. New York: Wiley.

BAYMAN, P., \& MAYER, R. E. (1984). Instructional manipulation of users' mental models of electronic calculators. International Journal of Man-Machine Studies, 20, 189-199.

BUSH, R. R., \& MOSTELLER, F. (1955). Stochastic models for learning New York: Wiley.

CATRAMBONE, R. (1994). Generalizing solution procedure learned from examples. Unpublished manuscript.

CATRAmbone, R. (1995). Aiding subgoal learning: Effects on transfer. Journal of Educational Psychology, 87, 5-17.

Catrambone, R., \& Holyoak, K. I. (1990). Learning subgoals and methods for solving probability problems. Memory \& Cognition, 18, 593-603.

DIXON, P., \& GABRYs, G. (1991). Learning to operate complex devices: Effects of conceptual and operational similarity. Human Factors, 33, $103-120$.

Halasz, F. G., \& Moran, T. P. (1983). Mental models and problem solving in using a calculator. In Proceedings of $\mathrm{CHI}$ '83: Human factors in computing sustems (pp. 212-216). New York: Association for Computing Machinery. 
Kamouri, A. L., Kamouri, J., \& Smith, K. H. (1986). Training by exploration: Facilitating the transfer of procedural knowledge through analogical reasoning. International Journal of Man-Machine Studies, 24, 171-192.

KIERAS, D. E., \& BovaIr, S. (1984). The role of a mental model in learning to operate a device. Cognitive Science, 8, 255-273.

Newell, A., \& Simon, H. A. (1972). Human problem solving. Englewood Cliffs, NJ: Prentice-Hall.

NoRman, M. F. (1964). Incremental learning on random trials. Journal of Mathematical Psychology, 1, 336-350.

Ross, B. H. (1984). Remindings and their effects in learning a cognitive skill. Cognitive Psychology, 16, 371-416.
Ross, B. H. (1987). This is like that: The use of earlier problems and the separation of similarity effects. Journal of Experimental Psychology: Learning, Memory, \& Cognition, 13, 629-639.

Ross, B. H. (1989). Distinguishing types of superficial similarities: Different effects on the access and use of earlier problems. Journal of Experimental Psychology: Learning, Memory, \& Cognition, 15, 456-468.

SMITH, E. E., \& GOODMAN, L. (1984). Understanding written instructions: The role of explanatory schema. Cognition \& Instruction, 1, 359-396.

(Manuscript received December 13, 1995; accepted for publication February 14, 1996.)

\section{Nominations for the Editorship of Psychonomic Bulletin \& Review}

Nominations are solicited for the editorship of Psychonomic Bulletin \& Review. The term of the present editor, Henry L. Roediger III, expires at the end of 1998. The new editor will begin an official 4- or 5-year term on January 1, 1999, and will begin to receive manuscripts early in January 1998. The Publications Committee of the Psychonomic Society expects to appoint the new editor by December 1997.

Nominations (including self-nominations) should be submitted by August 31, 1997, to:

Geoffrey Loftus

Chair, Psychonomic Bulletin \& Review Search Committee

Department of Psychology

University of Washington

Seattle, WA 98195 